\author{
P. U. Lohnstein ${ }^{1,4}$. J. Schipper ${ }^{1}$ A. Berlis ${ }^{2} \cdot$ N.-C. Gellrich ${ }^{3} \cdot$ W. Maier $^{4}$ \\ ${ }^{1}$ Hals-Nasen-Ohrenklinik, Universitätsklinikum Düsseldorf \\ ${ }^{2}$ Sektion Neuroradiologie, Neurochirurgische Universitätsklinik, \\ Universitätsklinikum Freiburg \\ ${ }^{3}$ Klinik für Mund-, Kiefer- und Gesichtschirurgie, Medizinische Hochschule Hannover \\ ${ }^{4}$ Universitätsklinik für Hals-, Nasen- und Ohrenheilkunde und Poliklinik, \\ Universitätsklinikum Freiburg
}

\title{
Posttraumatische Amaurose nach komplexer frontobasaler Fraktur
}

\section{Differenzialdiagnostische und therapeutische Aspekte}

Eine nach Schädel-Hirn-Trauma aufgetretene Amaurose wird generell als Indikation zur Einleitung der sofortigen klinischen und bildgebenden Diagnostik angesehen. Grundsätzlich kann die Amaurose durch eine direkte oder eine indirekte Sehnervenschädigung bedingt sein [28]. Während die erstgenannte Form mit einem radiologisch nachweisbaren Korrelat assoziiert ist (z. B. eingespießtes Knochenfragment, intraorbitales retrobulbäres Hämatom), fehlt dieses bei der letzteren.

Die therapeutischen Empfehlungen bei kanalikulärer postraumatischer Optikusneuropathie sind uneinheitlich. Unter den Kautelen einer evidenzbasierten Medizin ist die Datenlage hinsichtlich der Wertigkeit der besten therapeutischen Strategie unbefriedigend $[4,35,37]$. Einige Autoren empfehlen ein konservatives antiphlogistisches Vorgehen mit Kortikoiden [2, 14]. Von anderen Arbeitsgruppen wird ergänzend zu dieser antiödematösen Therapie eine chirurgische Dekompression favorisiert $[7,8,27]$ und mit der Notwendigkeit einer raschen Entlastung des N. opticus begründet, um die Integrität des Nervs zu erhalten und einer irreversiblen Neuronotmesis vorzubeugen. Diese Strategie, welche bereits 1973 von Lehnhardt empfohlen wurde [18], legt eine zeitnahe chirurgische Dekompression des N. opticus nahe $[8,16,21]$.

Jedoch ist vor chirurgischer Intervention zu bedenken, dass, bedingt durch die engen Nachbarschaftsbeziehungen in der Orbitaspitze, zusätzliche Traumata in dieser Region vorliegen können, die das Operationsrisiko erhöhen oder möglicherweise eine Kontraindikation zur Optikusdekompression darstellen können. In der vorliegenden Arbeit sollen anhand eines Fallberichts diesbezügliche Aspekte aufgegriffen und in ihren diagnostischen und therapeutischen Konsequenzen diskutiert werden.

\section{Fallbericht}

Die 42-jährige Patientin erlitt im Rahmen eines Fahrradsturzes ein Schädel-HirnTrauma (SHT) und wurde am Unfallort vom Notarzt wegen zunehmender Agitiertheit bei einem Glasgow-Coma-Scale von 11 intubiert. Die Schädelcomputertomographie (-CT) mit Weichteil- und Knochenfenster ergab intrakraniell eine Subarachnoidalblutung basal und links parietal ohne signifikantes Hirnödem. Da- neben lag eine Mittelgesichtsfraktur links vor mit Fraktur der Vorder- und Hinterwand des Sinus frontalis mit Hämatosinus, Sprengung der Sutura frontozygomatica links und Fraktur aller Orbitawände mit Nachweis eines ca. 5 mm großen knöchernen Fragments im Canalis nervi optici links und Frakturausstrahlung in den großen Keilbeinflügel links sowie den rechten Karotiskanal (• Abb. 1). Hinweise auf eine Fraktur des linken Karotiskanals bestanden nicht.

Nach Extubation wenige Stunden später gab die Patientin ein fehlendes Sehvermögen links an. Die augenärztliche Untersuchung ergab bei unauffälligem Befund rechts auf der linken Seite neben einem Lidhämatom eine komplette Afferenzstörung mit fehlender Pupillenreaktion bei Lichtreiz links sowie eine randscharfe und vitale Papille. Visuell evozierte Potenziale waren links nicht, rechts normal auslösbar. Weitere Symptome wie Exophthalmus oder Epistaxis lagen nicht vor.

Wir stellten die Indikation zur sofortigen transethmoidalen Dekompression des N. opticus links. Dieser Zugangsweg wurde gewählt, da er extrakraniell bleibt und eine Verlagerung des frisch traumatisierten Zerebrums vermeidet. Intra- 


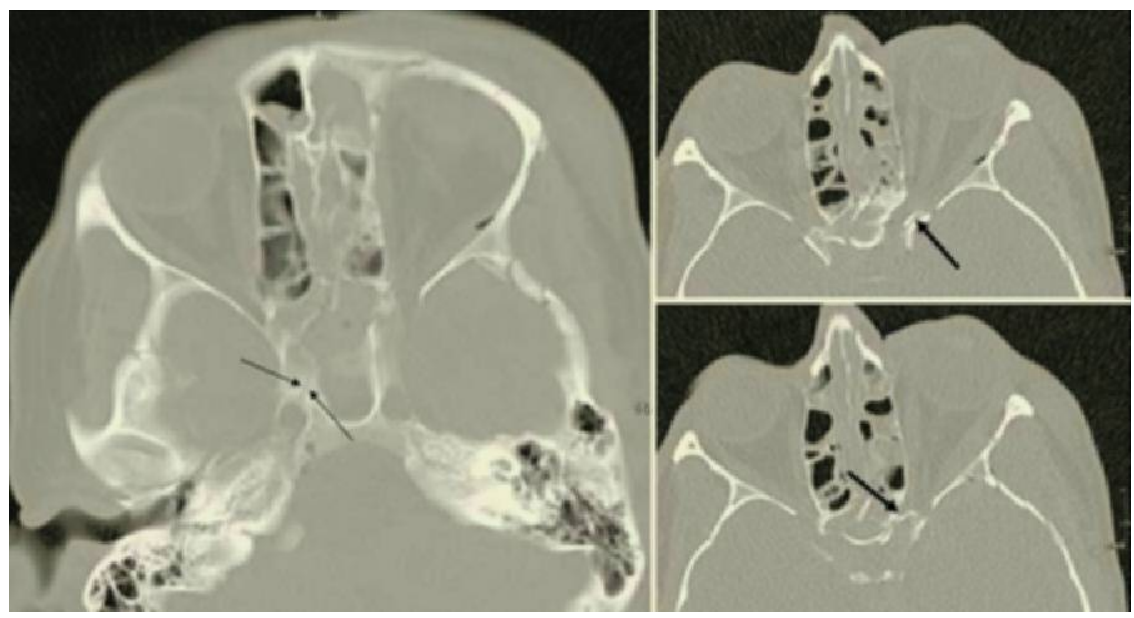

Abb. 1 A Die unmittelbar nach dem Schädel-Hirn-Trauma angefertigte CT zeigt eine Mittelgesichtsfraktur links mit Fraktur des Sinus frontalis und aller Orbitawände mit knöchernem Fragment im Canalis nervi optici links (dicker Pfeil) und Ausstrahlung in den großen Keilbeinflügel links sowie den rechten Karotiskanal (dünne Pfeile). Hinweise auf eine Fraktur des linken Karotiskanals bestanden nicht

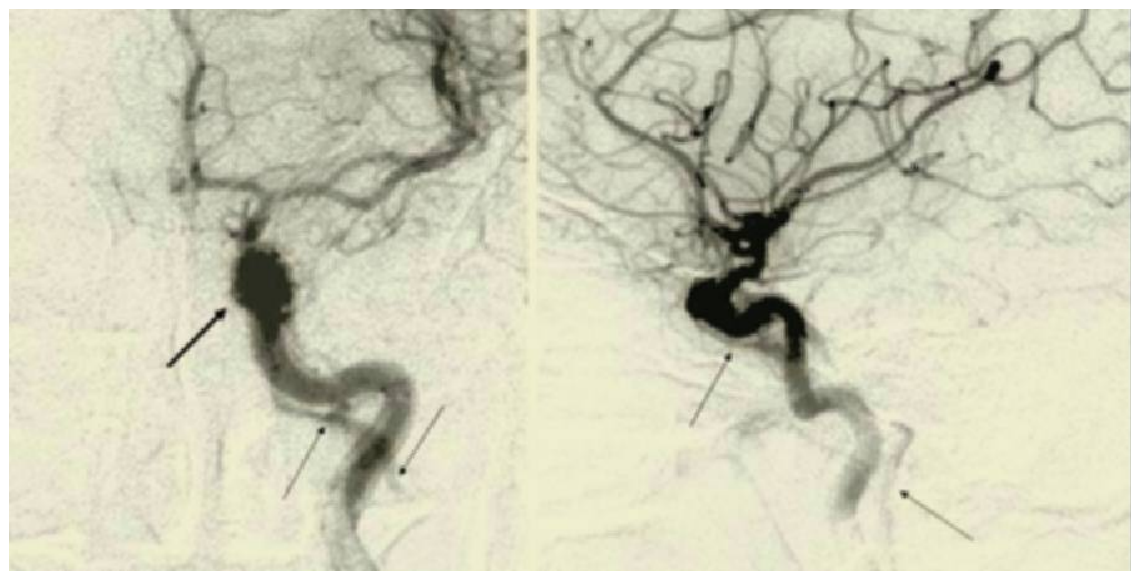

Abb. $2 \Delta$ Die zerebrale Angiographie stellt auf der linken Seite ein traumatisch bedingtes disseziertes Aneurysma der A. carotis interna im Siphon (dicker Pfeil) CCF Typ Barrow A mit Low-flow-Fistel (dünne Pfeile) zum Sinus cavernosus dar. Die A. ophthalmica kommt nicht zur Darstellung und ist verschlossen

operativ zeigte sich eine arterielle Blutung aus der rupturierten A. ethmoidalis anterior, die durch Koagulation gestillt wurde. Im hinteren Abschnitt der Lamina papyracea dorsal der A. ethmoidalis posterior und im Orbitatrichter bestanden eine ausgeprägte Mobilität und Hinweise auf eine Pulsation, sodass der Eingriff ohne Freilegung und Entfernung des Fragments beeendet wurde, um einer möglichen Blutung aus der A. carotis interna vorzubeugen.

Daraufhin wurde eine zerebrale Angiographie veranlasst. Diese ergab linksseitig ein traumatisch bedingtes disseziertes Aneurysma der A. carotis interna im Siphon, das sich bis nach intradural erstreckte, kombiniert mit einer traumatischen direkten Carotis-sinus-cavernosus-Fistel (CCF Typ Barrow A). Diese erscheint als Ursache für die intraoperativ aufgefallene Pulsation. Außerdem stellte sich die A. ophthalmica links verschlossen dar, welche im Bereich des dissezierten Aneurysmas entsprang (• Abb. 2).

Aufgrund der von extra- nach intradural reichenden Anseurysmaausdehnung bestand die Indikation zu einer endovaskulären Behandlung. Die optimale Therapie dieses dissezierten Aneurysmas mit irregulärer Morphologie bestand in einem Verschluss des Trägergefäßes (A. carotis interna), der nach Durchführung eines temporären Ballonokklusionstests sicher durchgeführt werden konnte. Der 2o-minütige Okklusionstest in Lokalanästhesie wurde ohne neurologische Defizite toleriert. Die deshalb durchgeführte erneute Angiographie zeigte, dass sich die CCF spontan verschlossen hatte (• Abb. 3).
In derselben Sitzung wurde konsekutiv in Vollnarkose die A. carotis interna proximal des Aneurysmas mit zwei ablösbaren Ballons verschlossen (• Abb. 4). Aufgrund der dissektionsbedingten hohen Vulnerabilität des Aneurysmas, und da aufgrund der Frontobasisfraktur ohnehin die Indikation zur neurochirurgischen Exploration mit frontobasaler Deckung bestand, wurde nach Abklingen des posttraumatischen Hirnödems die A. carotis interna distal des Aneurysmas über einen pterionalen Zugang mittels Clip operativ verschlossen (• Abb. 5). Introperativ fand sich der intrakanalikuläre Anteil des N. opticus gequetscht. Abgesehen von der persistierenden linksseitigen Amaurose war der postoperative Verlauf neurologisch bei dopplersonographisch nachweisbarem gutem Cross-flow über den Circulus arteriosus Willisi unauffällig, was durch eine Nachuntersuchung im Intervall bestätigt wurde.

\section{Diskussion}

Sehnervenschäden treten in etwa 2-5\% aller Schädel-Hirn-Traumata auf [10, 28]. Während bei direkten Nervenläsionen die Wahrscheinlichkeit einer spontanen Remission des Visus als minimal angesehen wird, wird sie bei indirekten Traumata im Schrifttum zwischen $20 \%$ und $40 \%$ eingestuft [36]. Ein bedeutender Prognosefaktor ist der initial-posttraumatische Visus: Bei absoluter Blindheit werden die Chancen einer Erholung, unabhängig von der Form der Therapie, als sehr gering beurteilt $[22,32]$.

In dem von uns beschriebenen Fall lag ein direktes Trauma vor, bedingt durch ein in den Canalis opticus disloziertes knöchernes Fragment. Direkte traumatische Läsionen des N. opticus können durch retrobulbäre Kompression, beispielsweise bedingt durch Einblutung aus einer rupturierten Ethmoidalarterie, durch Zerreißung des Nervs, oder durch Einspießung eines Fragments bedingt sein. Das Hämatom unterbricht durch intraorbitalen Druckanstieg die Mikrozirkulation des N. opticus und führt so zum ischämischen Funktionsverlust. Rupturen oder fragmentassoziierte Kompressionen des Nervs sind im Regelfall im kanalikulären Segment lokalisiert, da hier die geringsten 
Ausweichmöglichkeiten für den Nerv bestehen $[8,10,32]$. Er ist hier bindegewebig mit der Kanalwand verbunden, sodass bei knöchernen Verschiebungen ein hohes Verletzungsrisiko besteht [1].

Das Trauma induziert eine Kaskade chemischer Vorgänge, die zu sekundärem Vasospasmus und Ödem mit konsekutiver intraneuraler Kompression analog zu einem Kompartmentsyndrom führen [29]. Auch indirekte Traumata sind meist im kanalikulären Segment lokalisiert, da sich bei stumpfen Schädel-Hirn-Traumata hier auf die Frontobasis einwirkende Kräfte konzentrieren und zu Mikroläsionen des fest an den Kanal adhärenten Nerv führen können [11, 26]. Dementsprechend sind radiologische Nachweise einer Läsion im Verlauf des N. opticus selten und nur mittels Kernspintomographie und der Darstellung eines Optikusödems nachweisbar. Der Visusverlust wird auf Mikrotraumata zurückgeführt, die letztlich die genannte Kaskade auslösen, daneben auf Mikroläsionen der Axone [12]. Weiterhin kann eine Narbenbildung zur sekundären Kompression der Sehnerven führen [12].

Ein retrobulbäres Hämatom mit Sehnervenkompression wird im Schrifttum einheitlich als Indikation zur sofortigen operativen Entlastung angesehen $[4,8$, 9, 35]. Intrakanalikuläre Verletzungen werden demgegenüber nicht übereinstimmend beurteilt. Prospektive Studien konnte keine Überlegenheit der operativen Kanaldekompression über die medikamentöse Dekompression mit Kortikoiden belegen $[5,20,24]$.

Dies wird durch eine aktuelle Metaanalyse unter evidenzbasierten Kautelen bekräftigt [37]. Zudem fällt die Beurteilung, welche Patienten am ehesten von einer operativen Intervention profitieren, uneinheitlich aus: einige Autoren berichten, dass der geringste Nutzen bei $\mathrm{Pa}$ tienten mit einem massiven posttraumatischen Visusverlust resultiere [22], andere sehen gerade in dieser Patientengruppe die Operationsindikation als gegeben an, da die Kortisontherapie aufgrund der Schwere des Traumas nicht ausreiche [16, 25]. Im neueren Schrifttum wird jedoch auch über bessere Ergebnisse hinsichtlich des Visus bei Patienten mit operativer und medikamentöser Dekompression als

\section{P. U. Lohnstein · J. Schipper · A. Berlis · N.-C. Gellrich · W. Maier Posttraumatische Amaurose nach komplexer frontobasaler Fraktur. Differenzialdiagnostische und therapeutische Aspekte}

Zusammenfassung

Hintergrund. Nachdem über Jahrzehnte bei posttraumatischer Visusminderung mit Optikuskompression im knöchernen Kanal die Indikationsstellung zur chirurgischen Sehnervendekompression kontrovers diskutiert wurde, favorisiert die neuere Literatur überwiegend diesen Eingriff in Ergänzung zur hochdosierten Kortisontherapie. Das Risiko operationsbedingter Nebenwirkungen wird im Schrifttum meist als gering eingestuft.

Fallbericht. Wir berichten über eine Patientin, bei der im Rahmen eines Schädel-Hirn-

Traumas eine Fraktur des Canalis opticus links mit einseitigem Visusverlust auftrat. Bei der dekomprimierenden Operation bestand aufgrund der intraoperativen Symptomatik der Verdacht auf eine computertomographisch nicht nachweisbare AV-Fistel. Daher wurde der Eingriff unterbrochen. Die angeschlossene Angiographie zeigte neben einer Carotis-sinus-cavernosus-Fistel (CCF Typ Barrow A) ein disseziertes Aneurysma am Abgang der verschlossenen $A$. ophthalmica.

Diskussion und Schlussfolgerungen. Bei der Indikationsstellung zu einer operativen

\section{Posttraumatic amaurosis after complex frontobasal fracture. Differential diagnosis and therapy}

\section{Abstract}

Background. Whether surgical nerve decompression is indicated for the treatment of posttraumatic reduced vision with optic nerve compression in the bony canal has been a subject of controversy for decades. On balance, the recent literature suggests that this procedure is indeed indicated, as a supplement to high-dosed cortisone therapy. The risk of surgery-related side effects is usually rated low in the literature.

Case Report. We report on a woman patient in whom craniocerebral trauma involved a fracture of the left optic canal with unilateral loss of vision. In the decompression operation, intraoperative symptoms gave rise to the suspicion of an arteriovenous fistula, which had not been revealed by computer tomography and which was seen as sufficient grounds for discontinuing the procedure. In addition to a carotid artery-sinus cavernosus fistula (CCF Barrow type A), subsequent angiography revealed a dissected aneurysm at the branching of the occluded ophthalmic artery.
Entlastung des Canalis opticus nach traumatisch bedingter Visusminderung müssen potenzielle Begleitläsionen in der Nachbarschaft der Orbitaspitze und des Optikuskanals in Betracht gezogen werden. Nicht immer sind diese computertomographisch nachweisbar. Die Indikationsstellung zur chirurgischen Sehnervendekompression sollte in jedem Einzelfall kritisch abgewogen werden, ggf. sind hierzu weitere bildgebende Untersuchungen wie MR-Angiographie, CT-Angiographie oder eine konventionelle Angiographie erforderlich. Die Möglichkeiten und Indikationen zur Bildgebung werden diskutiert. Keinesfalls kann der Eingriff, wie von manchen Autoren postuliert, als „minimal-invasiv“ eingestuft werden.

\section{Schlüisselwörter}

Traumatische Visusminderung · Optikuskanalfraktur · Traumatische Optikusläsion . Carotis-sinus-cavernosus-Fistel · Frontobasale Fraktur · Karotisaneurysma
Discussion and Conclusions. In decisions on whether surgical relief of pressure on the optic canal is indicated after trauma-related visual loss, the possibility of secondary lesions near the tip of the orbita and the optic canal must be taken into account. These are not always revealed by computer tomography. The indications should be critically weighed up in each individual case, with additional imaging examinations, such as MR-angiography, CTangiography, or conventional angiography, performed as needed. The options and indications for imaging are discussed. The procedure can by no means be rated as "minimally invasive", as is postulated by some authors.

\section{Keywords}

Traumatic loss of vision - Optic canal fracture Traumatic optic nerve lesion - Carotid artery-sinus cavernosus fistula - Frontobasal fracture $\cdot$ Carotid artery aneurysm 


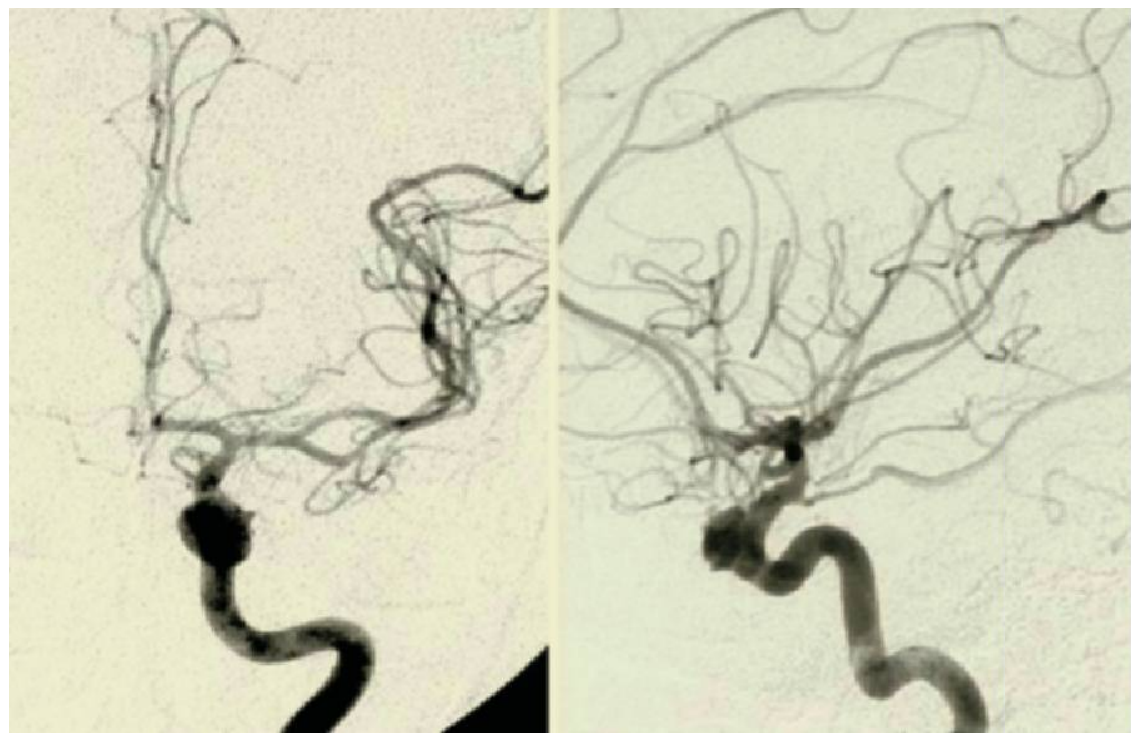

Abb. $3 \Delta$ Die zur endovaskulären Ausschaltung des Aneurysmas angefertigte Angiographie zeigt, dass sich die AV-Fistel zu diesem Zeitpunkt wenige Tage nach dem Trauma bereits spontan verschlossen hatte
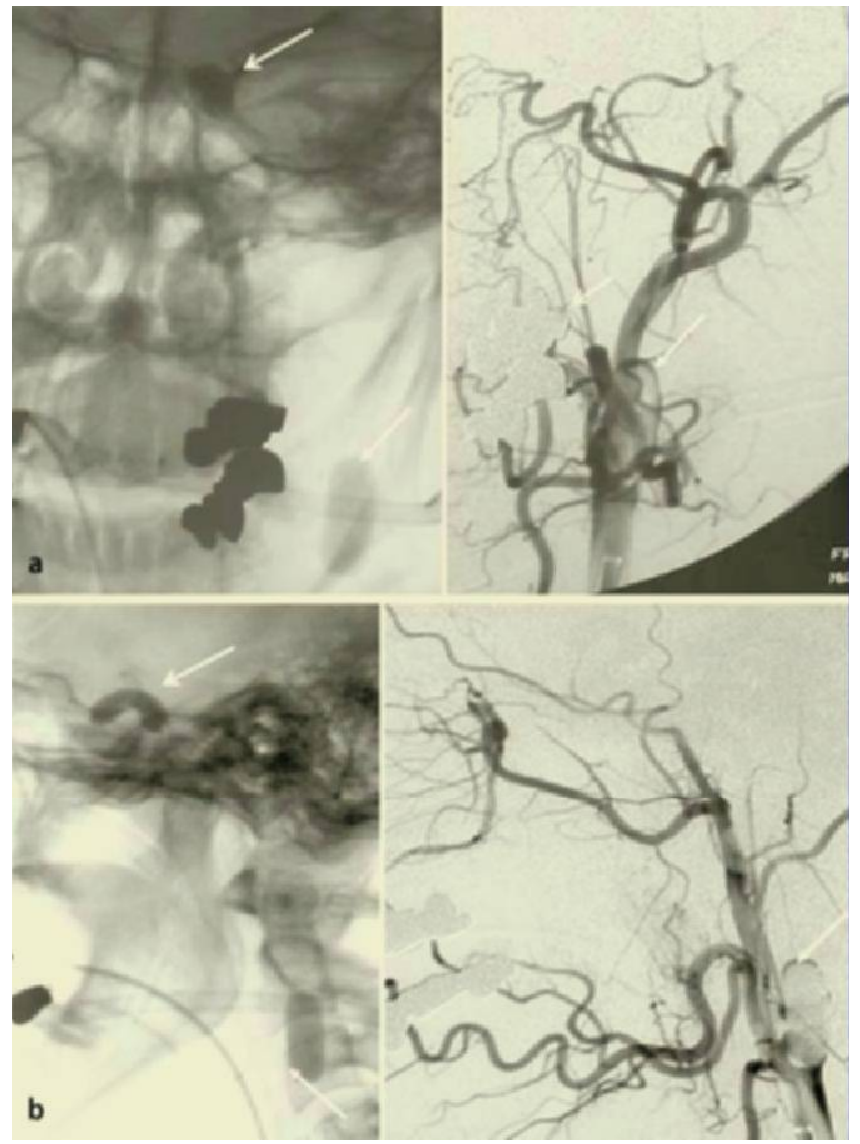

Abb. $4<$ Angiographische Abschlusskontrolle nach Platzierung der ablösbaren Ballons (Pfeile) in p.-a.(a) und seitlicher (b) Projektion

bei allein medikamentös behandelten $\mathrm{Pa}$ tienten berichtet $[16,21,22,25]$.

Entscheidet man sich zur Operation, so sind die engen Nachbarschaftsbeziehungen im Verlauf des Sehnervs unter topographisch-anatomischen Aspekten unbedingt zu beachten. Das Schrifttum for- die A. carotis interna. Die Schlitzung der Sehnervenscheide hingegen wird wegen des Risikos einer operationsbedingten Schädigung des Nervs oder der A. ophthalmica, einer Liquorrhö und Meningitis [7, 32] nicht empfohlen.

Verletzungen des knöchernen Kanals der A. carotis interna können im Rahmen eines Schädel-Hirn-Traumas mit oder ohne Verletzung des Gefäßes selbst auftreten. Eine Läsion des Karotiskanals ist im Dünnschicht-CT der Schädelbasis sichtbar, das notwendige Voraussetzung für eine Optikusdekompression ist, und kann eine Kontraindikation zur operativen Intervention darstellen [15]. Ein pulsierender Exophthalmus weist auf eine arteriovenöse Fistel hin und stellt die Indikation zur Angiographie vor jeglicher operativer Intervention in der Orbita und im Siebbein dar [8]. Jedoch besteht auch die Möglichkeit, dass eine Läsion der A. carotis interna ohne ipsilaterale knöcherne Begleitverletzung vorliegt.

In dem von uns beschriebenen Fall bestand eine nicht dislozierte Fraktur des Karotiskanals auf der nicht von einer Sehnervenverletzung betroffenen Seite mit Übergreifen auf die linke Keilbeinhöhle ventral des Karotiskanals, sodass der Kanal auf der erblindeten linken Seite intakt war. Erst die Angiographie konnte ein traumatisches Aneurysma darstellen, das im Bereich des Durchtritts des Gefäßes durch die Dura entstanden war und die A. ophthalmica miterfasst hatte. Tatsächlich entstehen Aneurysmen nicht nur durch direkte Gefäßverletzungen, sondern auch durch akzelerationsbedingte Scherkräfte [33], können also ohne simultane angrenzende Fraktur vorliegen.

Es handelt sich dabei um dissezierende Aneurysmen, die lediglich von Adventitia umgeben sind und dementsprechend ein erhebliches Blutungsrisiko besitzen. Ein traumatisches Aneurysma kann sich spontan zurückbilden, thrombosieren und mit der Gefahr einer thromboembolischen Komplikation einhergehen, oder sich ausweiten und rupturieren [33]. Bei intradural gelegenen Aneurysmen resultiert dann eine Subarachnoidalblutung, die in fast $60 \%$ aller Fälle auftritt und eine Letalität von 50\% aufweist [13]. Insgesamt werden in der Literatur Aneurysmen intrakranieller Gefäße, die 4,5\% aller leta- 
len Subarachnoidalblutungen zugrundeliegen [31], zumeist als endogene Formvariante beschrieben und nur selten auf eine exogen-traumatische Ursache zurückgeführt [31].

In der Schädel-CT unserer Patientin war eine frische Subarachnoidalblutung erkennbar, die vorwiegend über der Konvexität und im basalen Bereich nicht aneurysmanah nachzuweisen war, weil in diesem Falle das Aneurysma nicht rupturiert war.

Die CT- und MR-Angiographie können große Aneurysmen mit einer Sensitivität von über $90 \%$ abbilden, weisen bei einer Aneurysmagröße unter $3 \mathrm{~mm}$ aber nur eine Sensitivität von $61 \%$ bzw. $38 \%$ auf [34]. Deshalb ist die konventionelle Angiographie weiterhin der Goldstandard in der Diagnostik von Aneurysmen und bei einer Apoplexrate von unter 0,5\% in der Risiko-Nutzen-Abwägung gerechtfertigt [33]. Aufgrund seiner hohen Komplikationsrate ist beim traumatischen Aneurysma stets der zügige Verschluss desselben indiziert, was entweder endovaskulär oder operativ erfolgen kann. Die Therapie der Wahl ist hierbei normalerweise die Ausschaltung des betroffenen Gefäßsegments.

Eine gefäßerhaltende Therapie wird nur dann durchgeführt, wenn das Trägergefäß aufgrund einer schlechten oder fehlenden Kollateralversorgung nicht verschlossen werden kann [33]. So konnte in unserem Fall nach Durchführung eines temporären Ballonokklusionstests der Gefäßverschluss mit ablösbaren Ballons durchgeführt werden. Allerdings war lediglich ein proximaler Gefäßverschluss möglich, weil das Risiko einer manipulativ bedingten Aneurysmaruptur als hoch eingeschätzt wurde. Da aufgrund der frontobasalen Verletzung mit Durazerreißung ohnehin eine Deckung indiziert war, wurde im Rahmen dieses neurochirurgischen Eingriffs distal des Aneurysmas die A. carotis interna mit Hilfe eines Clips verschlossen.

Weiterhin bestand eine traumatische arteriovenöse (AV-)Fistel in den Sinus cavernosus, die entsprechend der Klassifikation nach Barrow als CCF Typ A einzuteilen war [3]. Ätiologisch muss von einer Dissektion der A. carotis interna im kavernösen Segment ausgegangen werden, wobei die Gefäßlazeration und der Einriss

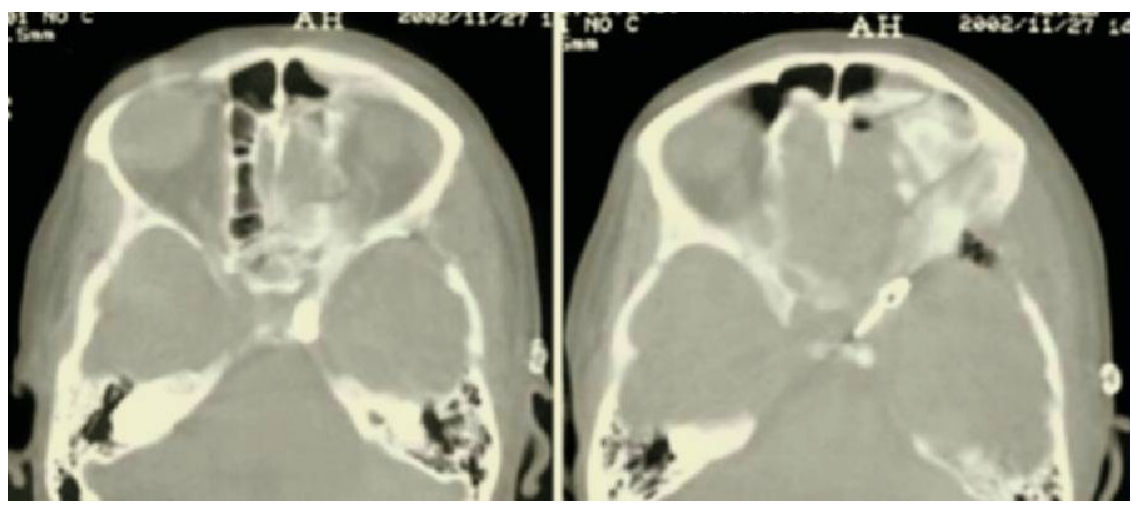

Abb. 5 D Die postprozedurale Schädel-CT bestätigt die korrekte Lage des distalen Ballons im Karotissiphon (links) sowie des neurochirurgisch platzierten Clips distal des dissezierten Aneurysmas (rechts)

des Durablatts zum Sinus cavernosus die Ursachen für die Fistelentstehung waren. Die Dissektion führte zum Entstehen des Aneurysmas, das durch Größenzunahme und Überschreiten der Dura nach intrakraniell mit einem proximalen Verschluss der A. ophthalmica einherging. Möglicherweise ist auch die Größenzunahme des Aneurysmas für den spontanen Verschluss der Fistel verantwortlich.

Von klinischer Bedeutung ist, dass die beschriebene Fistel ohne die klassischen Zeichen auftrat: Es bestand kein pulsierender Exophthalmus, weder eine orbitale oder okuläre Stauung, noch eine Augenmuskelparese oder ein retinales Hämatom, die gleichfalls als typisch bei Carotis-cavernosus-Fistel gelten [31]. Das Lidhämatom ist hingegen als unspezifisches Zeichen bei Orbitaverletzung zu werten. Das CT ist keine adäquate Methode der Darstellung eines Aneurysmas oder einer AV-Fistel [31], so waren diese auch in unserem präoperativen CT nicht darstellbar. Kleinere AV-Fisteln können auch der MR-tomographischen Darstellung inklusive konventioneller MR-Angiographie und dynamischer MR-Angiographie entgehen [31].

Der traumatisch bedingte Verschluss der A. ophthalmica könnte per se schon eine Amaurose durch Ischämie induzieren. Allerdings wird aufgrund der extrem guten Kollateralversorgung der A. ophthalmica über die A. facialis, über die Aa. ethmoidales aus der A. maxillaris und nicht selten auch über die A. meningea media ein proximaler Verschluss der A. ophthalmica sehr gut toleriert und führt in der Regel nur dann zur Erblindung, wenn der Verschluss nach distal reicht. So bewirkt eine Dissektion der A. carotis in- terna mit konsekutivem thrombotischem Verschluss der A. ophthalmica häufig eine Amaurosis fugax mit in Abhängigkeit von der Kollateralversorgung interindividuell unterschiedlicher Restitution des Visus [6] und nur selten einen vollständigen Visusverlust [23].

Es stellt sich in Anbetracht dieser potenziell eine frontobasale Fraktur begleitenden Traumafolgen die Frage, welche bildgebende Diagnostik bei einem Patienten mit Trauma des Optikuskanals vorzunehmen ist, bevor eine operative Dekompression erfolgt. Gellrich [8] sieht bei klinischen Symptomen einer Karotisverletzung, die sich in drückenden und pulsierenden Zephalgien, dem pulsierenden Exophthalmus durch AV-Fistel oder einer heftigen Epistaxis äußern kann, die Indikation zur Angiographie als gegeben an, trotz des dadurch bedingten Zeitverlustes bis zur Nervendekompression, da bei Vorliegen einer Karotisläsion ein hohes Operationsrisiko besteht. Zur Verringerung operativer Risiken empfiehlt er die Anwendung von Navigationstechniken. Diese sind aber bei Vorliegen einer vaskulären Läsion nicht unbedingt hilfreich.

Gibt es klinische Hinweise auf eine Karotisverletzung, auch ohne dass man einen direkten bildmorphologischen Hinweis in der Dünnschicht-CT hat, muss die Diagnostik erweitert werden. In diesen Fällen sollte stets zum Ausschluss einer traumatisch bedingten Gefäßverletzung eine angiographische Abklärung erfolgen. Wir bevorzugen meist die konventionelle Angiographie gegenüber der CToder MR-Angiographie, da sie wesentlich sensitiver ist.

Unser Fallbericht zeigt, dass grundsätzlich mit höchster operativer Vorsicht prä- 
pariert werden muss. Bestehen intraoperativ Hinweise auf eine Karotisverletzung, so ist es im Zweifel sinnvoller, den Eingriff vorzeitig zu beenden und neben der entsprechenden Diagnostik eine konservative Kortisontherapie vorzunehmen.

Keinesfalls können wir Aussagen im neueren Schrifttum [21, 25] unterstützen, wonach die Optikusdekompression, beispielsweise auf endonasalem Wege, ein minimal-invasiver Eingriff sei und die Patienten „wenig zu verlieren, aber viel zu gewinnen“ hätten. Vielmehr handelt es sich, wie unser Fall zeigt, um eine Operation mit nicht zu unterschätzender Morbidität, die einer sehr guten präoperativen Vorbereitung und höchster intraoperativer Sorgfalt mit einer guten Übersicht des anatomischen Situs bedarf. Dies impliziert, dass die alleinige endoskopische Sehnervendekompression nicht favorisiert werden sollte, sondern gerade bei schädelbasisverletzten Patienten allenfalls differenzialtherapeutisch Berücksichtigung finden kann. Die Indikationsstellung sollte in jedem Einzelfall unter Abwägung der patientenspezifischen $\mathrm{Be}$ funde und Symptome erfolgen.

\section{Fazit für die Praxis}

\begin{abstract}
Komplexe Traumen der Schädelbasis können aufgrund der unmittelbaren Nähe zur A. carotis interna zu deren Verletzung führen, ohne dass dies in klinischer oder computertomographischer Untersuchung deutlich wird. Eine operative Intervention in der Orbita und an der Schädelbasis sollte deshalb niemals ohne Dünnschicht-CT der Schädelbasis, sorgfältige Interpretation klinischer Befunde, welche auf Verletzungen der A. carotis interna hinweisen können, und ggf. weiterführende neuroradiologische Diagnostik vorgenommen werden! Auch sollte insbesondere bei intrakanalikulärer Optikusläsion eine sehr strenge Indikationsstellung zur Optikusdekompression erfolgen, da nach den Richtlinien der evidenzbasierten Medizin bisher ein positiver Effekt einer operativen Dekompression bei Traumata des Karotiskanals nicht eindeutig nachgewiesen werden konnte.
\end{abstract}

\section{Korrespondenzadresse}

\section{Prof. Dr. W. Maier}

Universitätsklinik für Hals-, Nasenund Ohrenheilkunde und Poliklinik Universitätsklinikum Freiburg Killianstraße 5, 79106 Freiburg maier@hno1.ukl.uni-freiburg.de

Interessenkonflikt. Es besteht kein Interessenkonflikt. Der korrespondierende Autor versichert, dass keine Verbindungen mit einer Firma, deren Produkt in dem Artikel genannt ist, oder einer Firma, die ein Konkurrenzprodukt vertreibt, bestehen. Die Präsentation des Themas ist unabhängig und die Darstellung der Inhalte produktneutral.

\section{Literatur}

1. Anand VK, Sherwood C, Al-Mefty O (1991) Optic nerve decompression via transethmoid and supraorbital approaches. Operative techniques in otolaryngology. Head Neck Surg 2: 157-166

2. Anderson RL, Panje WR, Gross CE (1982) Optic nerve blindness following blunt forehead trauma. Ophthalmology 89: 445-455

3. Barrow DL, Spector RH, Braun IF et al. (1985) Classification and treatment of spontaneous carotid cavernous fistulas. J Neurosurg 62: 248-256

4. Bloching M (2004) Indikationen und Technik der endonasalen Dekompression des N. opticus aus HNO-ärztlicher Sicht. Klin Monatsbl Augenheilkd 221: 927-932

5. Cook MW, Levin LA, Joseph MP (1996) Traumatic optic neuropathy. Arch Otolaryngol Head Neck Surg 122: 389-392

6. Eufinger $H$, Müller-Lung U, Krüger H (1998) Dissektion der A. carotis interna mit aufsteigender Thrombosierung der A. ophthtalmica als seltene Differenzialdiagnose der posttraumatischen Amaurose. Mund Kiefer Gesichts Chir 2: 209-212

7. Fukada Y (1975) Results in 300 cases of decompression of the optic nerve. Mod Probl Ophthalmol 14: 474-481

8. Gellrich NC (1999) Kontroversen und aktueller Stand der Therapie von Sehnervenschäden in der kraniofazialen Traumatologie und Chirurgie. Mund Kiefer Gesichts Chir 3: 176-194

9. Gerbino G, Ramieri GA, Nasi A (2005) Diagnosis and treatment of retrobulbar haematomas following blunt orbital trauma: a description of eight cases. Int J Oral Maxillofac Surg 34: 127-131

10. Gossmann MD, Roberts DM, Barr CC (1992) Ophthalmic aspects of orbital injury - a comprehensive diagnostic and management approach. Clin Plastic Surg 19: 71-85

11. Gross CE, DeKock JR, Panje WR (1981) Evidence for orbital deformation that may contribute to monocular blindness following minor frontal head trauma. J Neurosurg 55: 963-966

12. Hager G, Gerhardt HJ, Maruniak M (1975) Indikationen und Ergebnisse operativer Freilegung traumatisch geschädigter Sehnerven. Klin Monatsbl Augenheilkd 167: 515-526

13. Holmes B, Harbaugh RE (1993) Traumatic intracranial aneurysms: a contemporary review. J Trauma 35: 855-860

14. Hsieh CH, Kuo YR, Hung HC (2004) Indirect traumatic optic neuropathy complicated with periorbital facial bone fracture. J Trauma 96: 795-801

15. Joseph M, Lessel S, Rizzo J (1999) Extracranial optic nerve decompression for traumatic optic neuropathy. Arch Ophthalmol 108: 1091-1093
16. Kountakis SE, Maillard AAJ, El-Harazi SM et al. (2000) Endoscopic optic nerve decompression for traumatic blindness. Otolaryngol Head Neck Surg 123: 34-37

17. Lang J (1988) Über die Cellulae ethmoidales posteriores und ihre Beziehungen zum Canalis opticus. HNO 36: 49-53

18. Lehnhardt E (1973) Die Dekompression des Nervus opticus bei Fraktur der Rhinobasis. HNO 21: 158160

19. Lentrodt J, Unsöld R, Bosche J (1991) Amaurose nach operativer Versorgung von Orbitabodenfrakturen - eine unvorhersehbare Komplikation? Fortschr Kiefer Gesichtschir 36: 150-151

20. Levin LA, Beck RW, Joseph MP (1999) The treatment of traumatic optic neuropathy. Ophthalmology 106: 1268-1277

21. Maurer J, Hinni M, Mann W, Pfeiffer N (1999) Optic nerve decompression in trauma and tumour patients. Eur Arch Otorhinolaryngol 256: 341-345

22. Mine S, Yamakami I, Yamaura A et al. (1999) Outcome of traumatic optic neuropathy. Comparison between surgical and nonsurgical treatment. Acta Neurochir 141: 27-30

23. Neudecker S, Bau V, Behrmann C et al. (2004) Unilaterale Amaurose als einziges fokales Zeichen einer Carotis-communis-Dissektion. Klin Monatsbl Augenheilkd 221: 509-512

24. Perry JD (2004) Treatment of traumatic optic neuropathy remains controversial. Arch Otolaryngol Head Neck Surg 130: 1000

25. Rajiniganth MG, Gupta AK, Gupta A, Bapuraj JR (2003) Traumatic optic neuropathy. Visual outcome following combined therapy protocol. Arch Otolaryngol Head Neck Surg 129: 1203-1206

26. Rochels R (1990) Holographic deformation analysis of the optic canal in blunt cranial trauma. Fortschr Ophthalmol 87: 182-185

27. Schmidbauer JM, Müller E, Höh H (1998) Transsphenoidale Frühdekompression bei indirekter traumatischer Optikusneuropathie. HNO 46: 152 156

28. Sofferman RA, Burlington VT (1981) Sphenoethmoid approach to the optic nerve. Laryngoscope 91: 184-196

29. Steinsapir KD, Goldberg RA (1994) Traumatic optic neuropathy. Surv Opohthalmol 38: 487-518

30. Steinsapir KD, Seiff SR, Goldberg RA (2002) Traumatic optic neuropathy: where do we stand. Opthal Plast Reconstr Surg 18: 232-234

31. Szikora I (2004) Dural arteriovenous malformations. In: Forsting M, Baert AL, Sartor K (Eds.) Intracranial vascular malformations and aneurysms. Springer, Berlin Heidelberg New York Tokio, pp 101-141

32. Thakar A, Mahapatra AK, Tandon DA (2003) Delayed optic nerve decompression for indirect optic nerve injury. Laryngoscope 113: 112-119

33. Wanke I, Dörfler A, Forsting M (2004) Dural arteriovenous malformations. In: Forsting M, Baert AL, Sartor K (eds) Intracranial vascular malformations and aneurysms. Springer, Berlin Heidelberg New York Tokio, pp 143-247

34. White PM, Wardlaw JM, Easton V (2000) Can noninvasive imaging accurately depict intracranial aneurysms? A systematic review. Radiology 217: 361370

35. Wilhelm H (2004) Traumatische Optikusneuropathie - der aktuelle Stand. Klin Monatsbl Augenheilkd 221: 702-705

36. Yang WG, Chen CT, Tsai PK et al. (2004) Outcome for traumatic neuropathy - surgical versus nonsurgical treatment. Ann Plast Surg 52: 36-42

37. Yu Wai Man P, Griffiths PG (2005) Surgery for traumatic optic neuropathy. Cochrane Database Syst Rev 4: CD005024 